\title{
III.13 Compatibility of ZDDP and Ionic Liquid Anti-Wear Additives with Hard Coatings for Engine Lubrications
}

\section{Overall Objectives}

- Investigate the compatibility of engine lubricant antiwear (AW) additives, specifically conventional zinc dialkyldithiophosphate (ZDDP) and newly developed ionic liquids (ILs), with selected commercial hard coatings

- Provide fundamental understanding to guide future development of engine lubricants

\section{Fiscal Year (FY) 2015 Objectives}

- Further understand the tribochemical interactions between AW additives and non-metallic coatings in boundary lubrication regime, and investigate the AW coating compatibility in mixed lubrication regime

- Reveal the wear process and tribofilm formation via wear debris characterization

\section{FY 2015 Accomplishments}

- Oak Ridge National Laboratiry Significant Event Award

- Five journal papers

- Five conference presentations

- New wear mechanism discovered for the excessive material removal on the steel counterface against diamond-like carbon (DLC) coating, DLC-catalyzed tribocorrosion

- Evolution of wear debris generation revealed and correlated to the wear process and tribofilm formation

\section{Future Directions}

This task of AW coating compatibility has successfully been concluded and we are shifting the efforts to investigate the compatibility between AW additives and non-ferrous engine bearing alloys, specifically bronze and aluminum alloys, in FY 2016.

\section{Introduction}

Current engine lubricants and their additive packages were designed for ferrous alloy bearing surfaces. The compatibility between oil AW additives and non-metallic hard coatings is little known. Fundamental understandings gained in this study will help guide future development

\author{
Jun Qu (Primary Contact), Yan Zhou, \\ Donovan N. Leonard, Harry M. Meyer, \\ and Huimin Luo \\ Oak Ridge National Laboratory \\ Materials Science \& Technology Division \\ P.O. Box 2008 \\ Oak Ridge, TN 37831-6063 \\ Phone: (865) 576-9304 \\ Email: qujn@ornl.gov \\ DOE Technology Development Manager: \\ Kevin Stork \\ Subcontractor: \\ Prof. Seong H. Kim \\ Pennsylvania State University \\ University Park, PA 16802
}

of engine lubricants. A synergistic lubricant-coating combination will potentially maximize the benefits in fuel economy.

\section{Approach}

We experimentally studied the friction and wear behavior for selected non-metallic hard coatings lubricated by selected AW additives using tribological bench tests in well-defined conditions. The tribochemical interactions between the AW additives and the coating surfaces were investigated via comprehensive tribofilm characterization.

\section{Results}

\section{Investigate the Compatibility between AW and Hard Coatings}

In FY 2014, we had reported a significantly increased wear on the steel ball that rubbed against a hydrogenated DLC (a-C:H) coating in a base oil containing 1\% ZDDP or phosphonium-organophosphate IL (IL-A), as shown in Figure $1 \mathrm{~b}$ [1]. In contrast, a boride coating, $\mathrm{AlMgB}_{14^{-}}$ $\mathrm{TiB}_{2}$, was tested in the same condition as that for a-C:H DLC, but showed no detrimental impact on the steel ball. In FY 2015, we further explored the increased wear phenomenon for the steel ball rubbing against a nonhydrogenated DLC coating (ta-C DLC). A similar trend 
was observed; the addition of $1 \%$ ZDDP into the base oil resulted in an $80 \%$ increase in the ball wear. On the other hand, when $1 \%$ of a non-phosphate IL, phosphoniumcarboxylate (IL-B, Figure 1a), was used instead, no such wear increase was occurred on the steel ball (see Figure 1b) [1]. Results clearly indicate incompatibility between phosphate-based AW additives and DLC coatings.

Here we propose a new mechanism for such excessive wear of the steel ball when rubbing against DLC; DLC catalyzes tribochemical reactions between the phosphate AW additives and the steel ball surface, causing tribocorrosion. Both sp2 C [2] and sp3 C [3] have been reported with catalytic effects. Without catalysis, the phosphate-based AW additives (ZDDP or IL) form a dense, protective tribofilm on the steel surface as normally observed. However, in presence of sp2 and/or sp3 C, tribochemical reactions would occur at an unacceptably high rate, as illustrated in Figure 1c, which is expected to generate a porous tribofilm. If no AW, the compressed wear particles are likely to be removed from the surface due to poor bonding. Further illustrated in Figure 2a,

a)

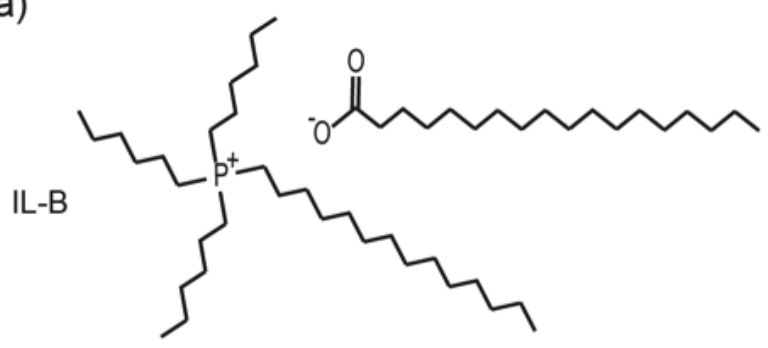

c)

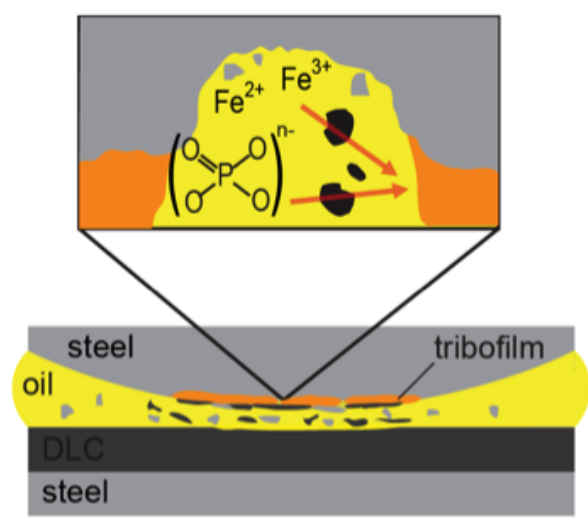

when an AW is present, the wear particles react with AW molecules and transform to a dense protective layer. When tribocorrosion occurs, a primitive, porous tribofilm containing larger debris is expected to form. Figure $2 \mathrm{~b}$ presents the transmission electron microscope cross-sectional images of two tribofilms on the steel ball against the DLC and steel flats, respectively. The tribofilm on the ball against steel has a double layer structure containing a top porous layer of fine nanoparticles and a bottom dense amorphous layer. In contrast, the tribofilm on the ball against DLC contains only a porous layer of nanoparticles, which supports our hypothesis of tribocorrosion [1].

Stribeck curves were generated using a steel cylinder sliding against steel, DLC (a-C:H), and $\mathrm{TiB}_{2}$ flats in polyalphaolefin (PAO) $4 \mathrm{cSt}$ base oil, PAO + 1\% ZDDP, and $\mathrm{PAO}+1 \%$ IL by using a variable load-speed bearing tester. DLC exhibited little changes between cycles, while the $\mathrm{TiB}_{2}$ surface, on the other hand, produced a lower friction after running-in. The $\mathrm{TiB}_{2}$ flat surface was observed with some material transferred from the steel

b)
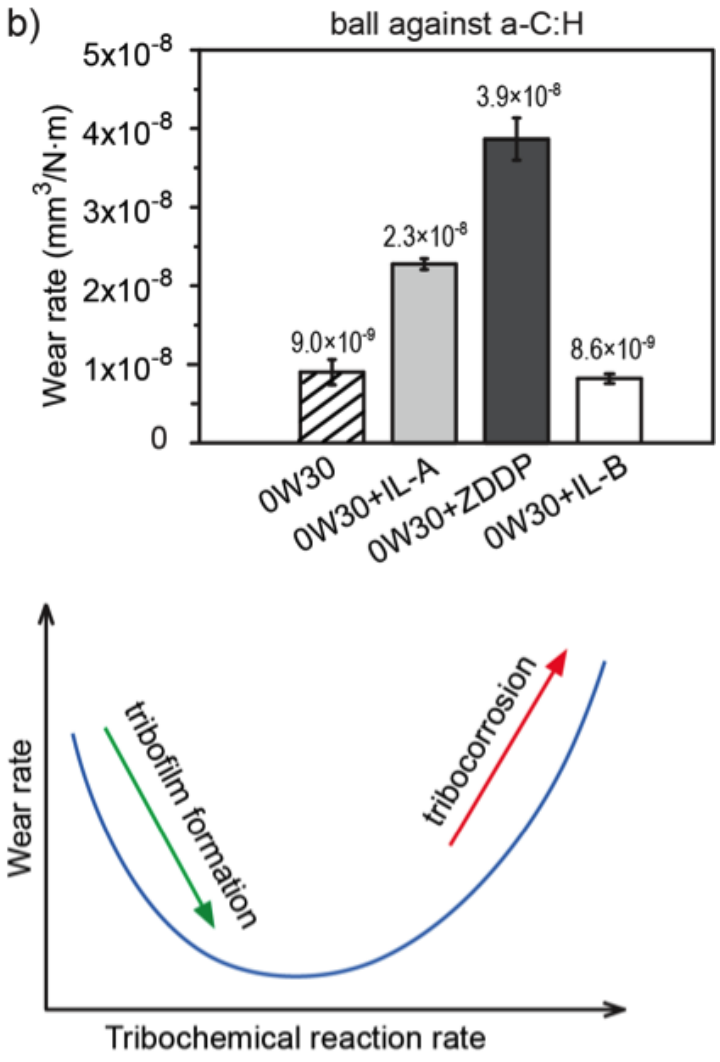

Figure 1. Unlike ZDDP or phosphonium-phosphate IL (IL-A), phosphonium-carboxylate IL (IL-B) does not cause increased wear on the steel ball against DLC. (a) Molecular structure of IL-B. (b) Wear rates of the steel balls against a-C:H DLC in oils containing three different additives. (c) Proposed mechanism: phosphate additives (ZDDP or IL-A) normally react with the steel ball surface to form a protective tribofilm; however, when catalyzed by sp2 and/or sp3 C, tribochemical reactions occur at an unacceptably high rate to cause rapid material removal [1]. 
a

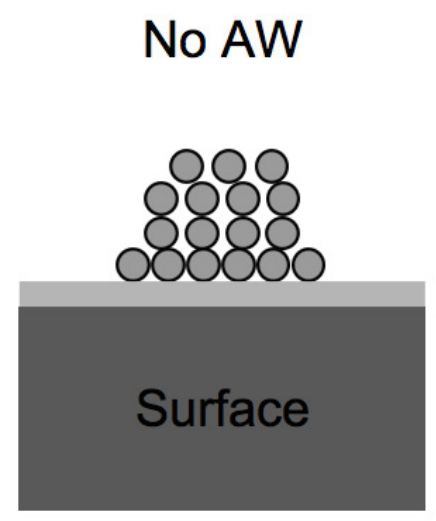

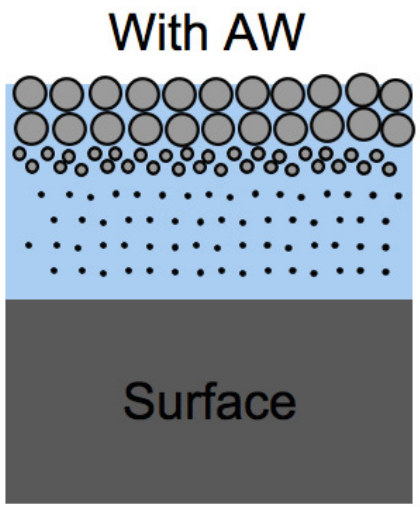

\section{With AW and catalyst}

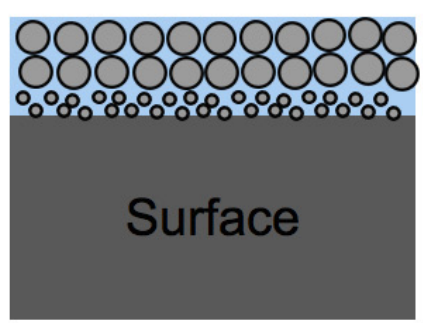

b

ball against steel in $0 \mathrm{~W} 30+\mathrm{IL}$

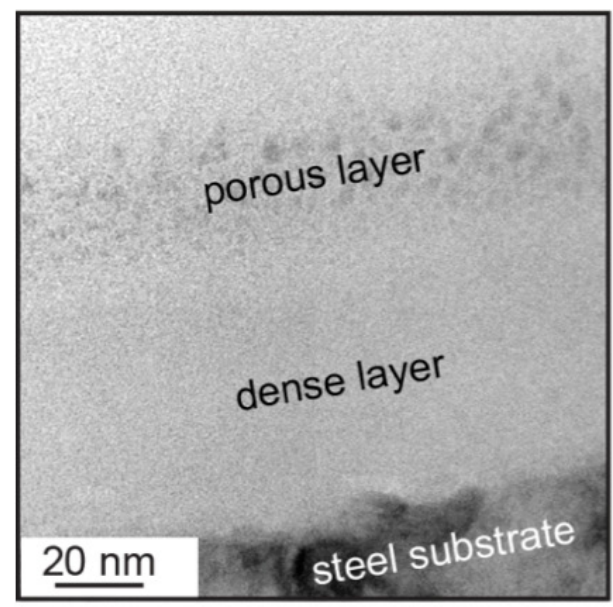

ball against a-C:H in $0 \mathrm{~W} 30+\mathrm{IL}$

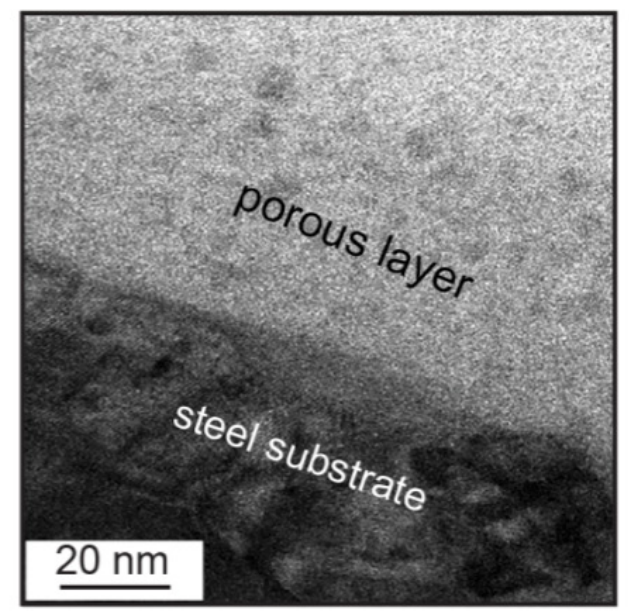

Figure 2. (a) Schematic representation of tribofilms (not in scale) under different conditions. (b) Cross-sectional bright field transmission electron microscopy images of the sample lubricated by OW30+IL-A trihexyltetradecylphosphonium bis(2-ethylhexyl) phosphate ([P $\left.\left.\mathrm{P}_{66614}\right][\mathrm{DEHP}]\right)$.

ball, but there is neither measurable material loss nor transfer on the DLC flat.

\section{Reveal Wear Debris Evolution to Correlate with Wear Process and Tribofilm Formation}

Figure 3 a shows the scanning electron microscope images of the wear scar of the steel ball. The worn surface shows three distinctive regions. Region 1 is relatively smooth and takes the most of the wear scar, and Region 2 is rather rough. Region 3 is a stripe of dark patches that runs parallel to the direction of the sliding. The two-dimensional profile corresponds to the marked line after subtracting ball curvature and shows grooving and protrusion of Regions 2 and 3, respectively. In Figure 3b, focused ion beam-aided cross-sectional scanning transmission electron microscope (STEM) images illustrate an oxidation layer of $5 \mathrm{~nm}$ that has a continuous coverage of the surface of Regions 1 and 2. The patches of Region 1 are part of a discrete adhesive tribofilm with its thickness up to $250 \mathrm{~nm}$ with randomly embedded nanoparticles. The stresses encountered by the three regions and the difficulties of wear debris removal both are ranked as $2>3>1$. Region 1 is much smoother than the other two regions because of the little trapped wear debris and low contact stress. For Region 2, the highest contact stress worked collectively with the large amount of trapped wear debris, resulting in three-body abrasive wear that was accompanied by micro-scuffing. The high contact stress also reduced the longevity of adhered tribofilm, causing it to be removed swiftly and leaving only the natural oxidation layer of steel. For Region 3, wear debris particles were regularly removed while some of them were able to adhere to the surface, due to the medium contact stress. Cross-sectional energy dispersive $\mathrm{X}$-ray spectroscopy (EDS) elemental maps in Figure 3b confirm that the tribofilms formed in the base oil are mainly composed of iron oxides. 
a

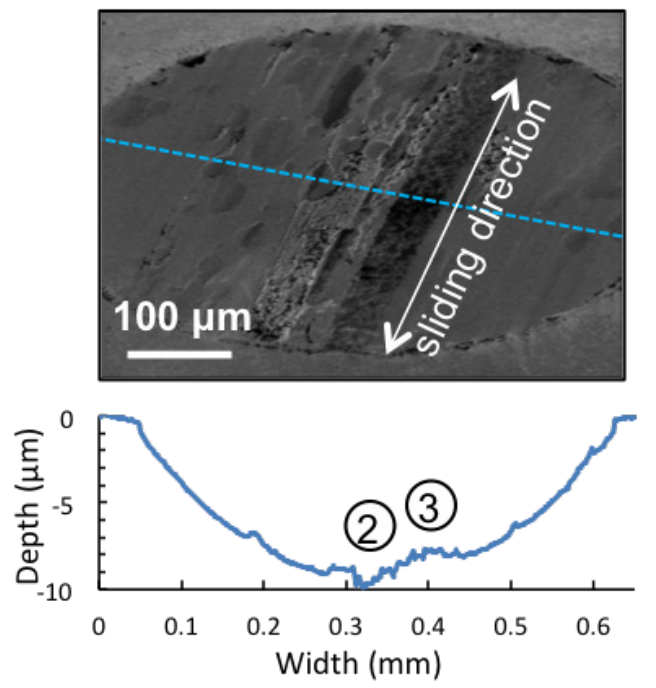

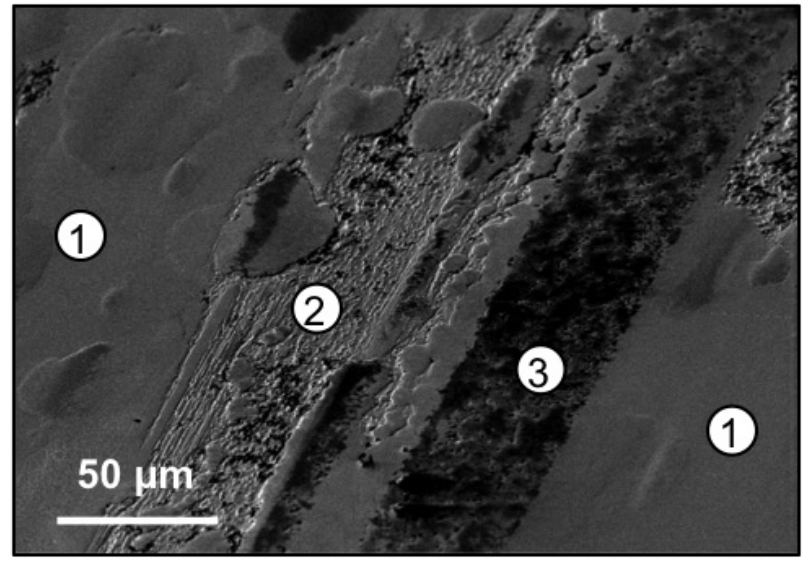

(1) b
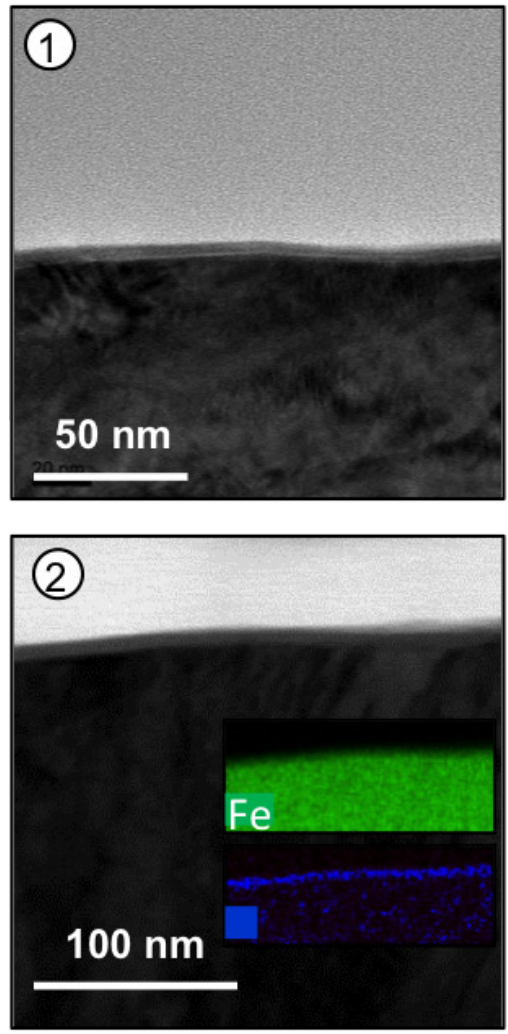
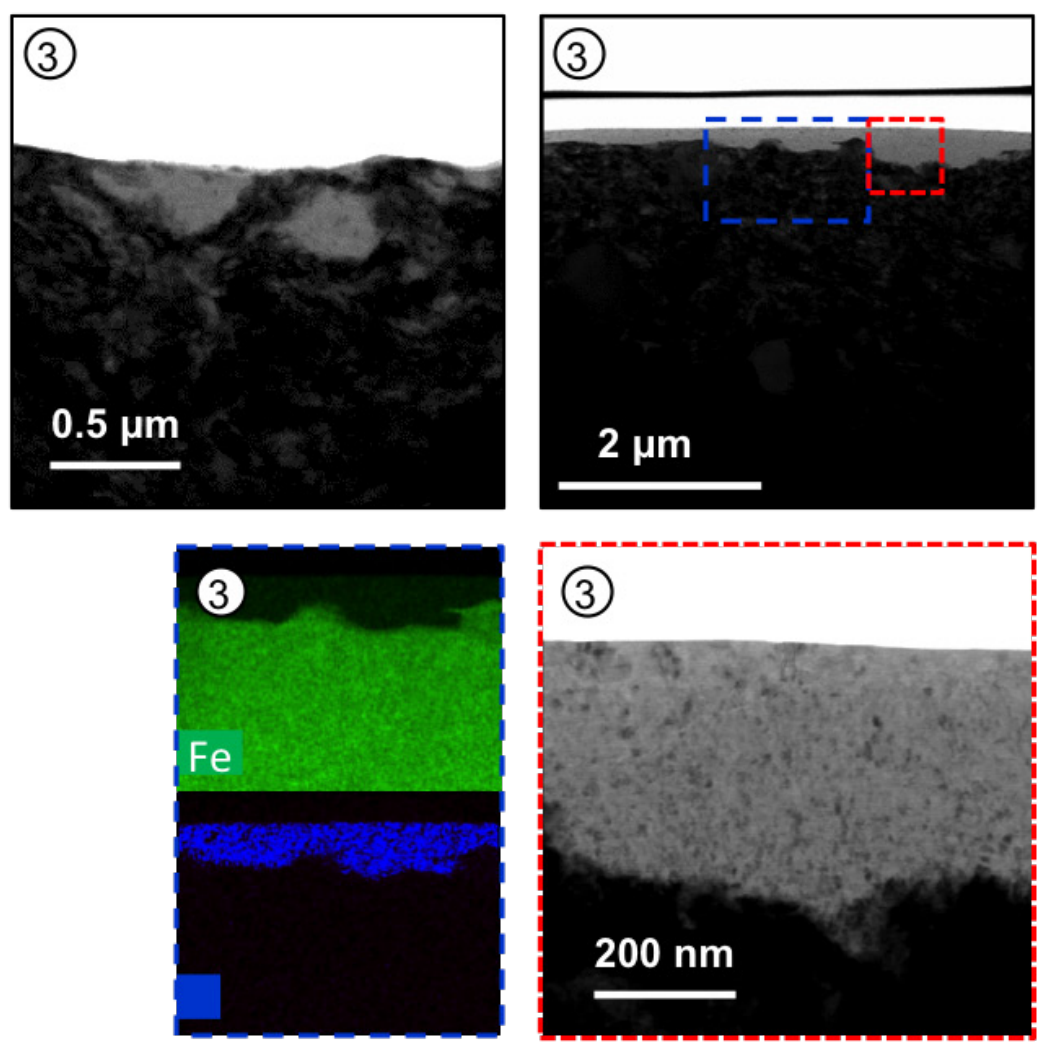

Figure 3. (a) Scanning electron microscope images of the wear scar on a steel ball against a steel flat lubricated by SAE 0W-30 base oil. (b) Cross-sectional STEM images and EDS elemental maps.

Wear debris analysis complements the characterization of worn surface and tribofilm. Figure $4 \mathrm{a}$ shows the STEM images of aggregates of wear debris particles from the steel-steel contact in 0W-30. Metallic clusters (magnified image) and spindle-like particles (indicated by the arrow) are scattered in the aggregate. The spindle-like particle contains Fe and $\mathrm{O}$, indicated by EDS mapping. The morphological change of wear debris reflects a dynamic and evolutionary process. The size of wear particles is inversely proportional to the received energy during sliding, and the big wear particles are ground down during tribotests, as illustrated in Figure $4 \mathrm{~b}$. The collision between asperities generates wear debris particles of varied sizes. The grinding process removes edges and vortexes of debris particles and reduces their size. When the particles get small enough, the tribofilm would 


\section{a}
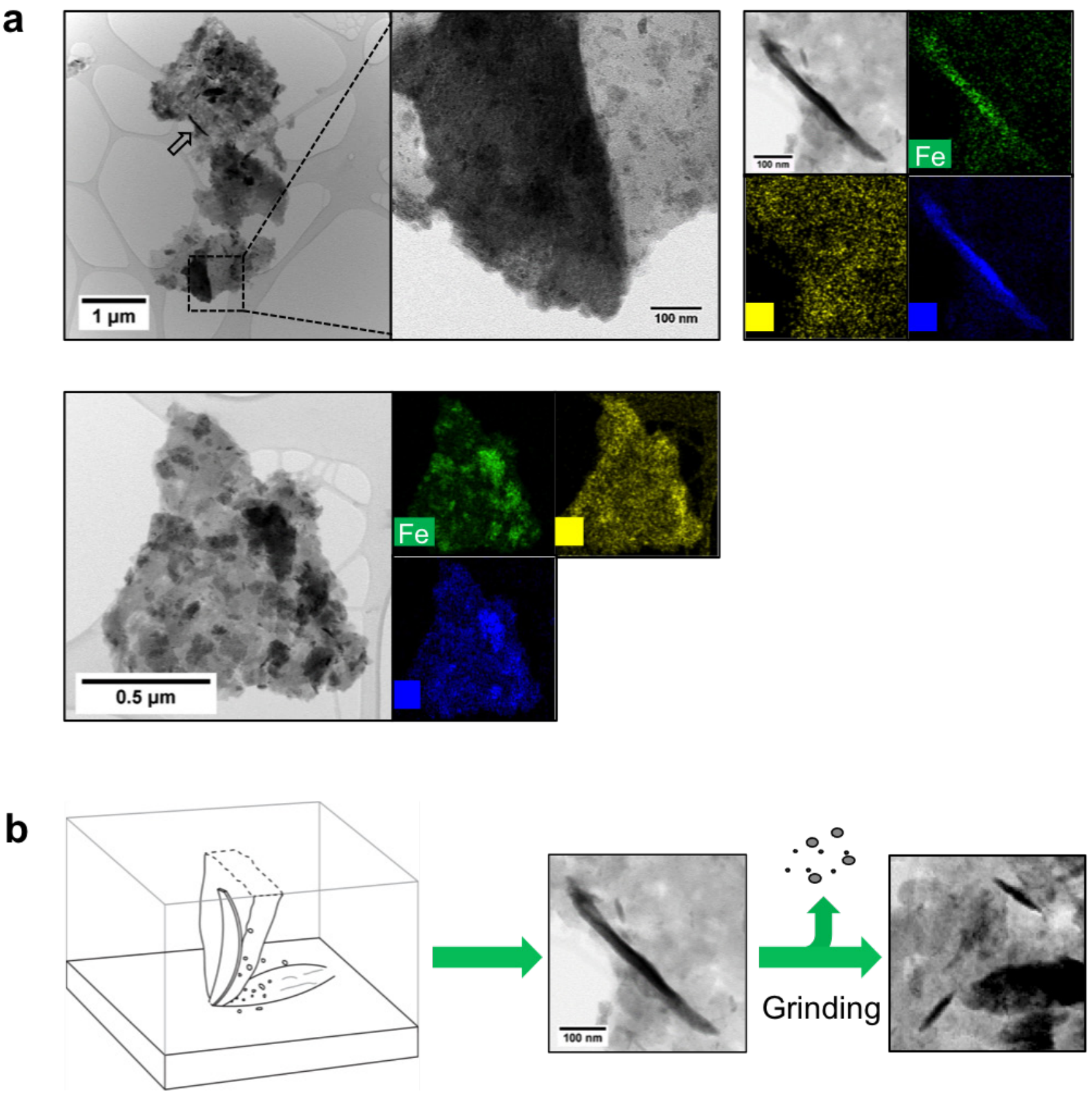

Figure 4. (a) STEM images and EDS elemental maps of the aggregates of wear particles from steel-steel contact in the base oil; (b) Evolution of wear debris from large to small with edges and vortexes lost in the process.

incorporate them by embedding with the matrix. When an $\mathrm{AW}$ is present at the interface, the AW molecules chemically react with the wear debris to incorporate and bind the reaction products and particles to form a protective tribofilm.

\section{Conclusions}

- A significantly increased wear rate was observed on a steel ball when it was rubbed against a DLC coating in the presence of an organophosphate AW additive. Such detrimental effects do not apply to non-carbon hard coatings or non-phosphate additives. The ball wear is proposed to be accelerated by DLC-catalyzed, high-rate tribochemical reactions with the phosphate additives.

- The compatibility results between non-metallic coatings and lubricant AW additives provide new insights for future development and implementation of advanced lubricants and materials. 
- The wear debris characterization deepens fundamental understanding if the wear process and the dynamics of tribofilm formation.

\section{References}

1. Y. Zhou, D.N. Leonard, H.M. Meyer, H. Luo, J. Qu, Advanced Materials Interfaces (2015) 2(15) DOI: 10.1002/admi.201500213.

2. H. Huang, J. Huang, Y.M. Liu, H.Y. He, Y. Cao, K.N. Fan, Green Chem 2012, 14, 930; H.L. Fei, R.Q. Ye, G.L. Ye, Y.J. Gong, Z.W. Peng, X.J. Fan, E.L.G. Samuel, P.M. Ajayan, J.M. Tour, Acs Nano 2014, 8, 10837.

3. Y. Cao, X. Luo, H. Yu, F. Peng, H. Wang, G. Ning, Catalysis Science \& Technology 2013, 3, 2654; D.S. Su, N. I. Maksimova, G. Mestl, V. L. Kuznetsov, V. Keller, R. Schlogl, N. Keller, Carbon 2007, 45, 2145.

\section{FY 2015 Publications/Presentations}

1. Y. Zhou, D.N. Leonard, H.M. Meyer, H. Luo, J. Qu, "Does the use of diamond-like carbon coating and organophosphate lubricant additive together causes excessive tribochemical material removal?" Advanced Materials Interfaces (2015) 2(15) DOI: 10.1002/ admi.201500213.

2. J. Qu, H.M. Meyer III, Z.-B. Cai, C. Ma, H. Luo, "Characterization of ZDDP and ionic liquid tribofilms on boride and DLC coatings," Wear 332-333 (2015) 1273-1285.

3. Z.-B. Cai, Y. Zhou, J. Qu, "Effect of oil temperature on tribological behavior of a lubricated steel-steel contact," Wear 332-333 (2015) 1158-1163.

4. Y. Zhou, J. Dyck, T. Graham, H. Luo, D.N. Leonard, J. Qu, "Ionic liquids composed of phosphonium cations and organophosphate, carboxylate, and sulfonate as lubricant anti-wear additives," Langmuir 30 (2014) 13301-13311.
5. Z.-B. Cai, H.M. Meyer III, C. Ma, M. Chi, H. Luo, J. Qu, "Comparison of the tribological behavior of steelsteel and $\mathrm{Si}_{3} \mathrm{~N}_{4}$-steel contacts in lubricants with ZDDP or ionic liquid," Wear 319 (2014) 172-183.

6. Y. Zhou, D. Leonard, H. Luo, J. Qu, "Characterization of wear debris generated in steel-steel and steel-DLC contacts lubricated by an ionic liquid additized oil," 2015 STLE Tribology Frontiers Conference, Denver, October 25-27, 2015.

7. Y. Zhou, H.M. Meyer III, D.N. Leonard, H. Luo, J. Qu, "Compatibility of ZDDP and ionic liquid with DLC and boride coatings," STLE 70th Annual Meeting, Dallas, May 17-21, 2015.

8. J. Qu, H.M. Meyer III, Z.-B. Cai, C. Ma, H. Luo, "Characterization of ZDDP and ionic liquid tribofilms on boride and DLC coatings," 20th International Conference on Wear of Materials, Toronto, Canada, April 12-16, 2015.

9. Z.-B. Cai, Y. Zhou, J. Qu, "Effect of oil temperature on tribological behavior of a lubricated steel-steel contact," 20th International Conference on Wear of Materials, Toronto, Canada, April 12-16, 2015.

10. J. Qu, H.M. Meyer III, Y. Zhou, Z.-B. Cai, C. Ma, H. Luo, "Tribofilm Characterization for Understanding the Compatibility Between Lubricant Anti-Wear Additives and Non-Metallic Coatings," 2014 STLE Tribology Frontiers Conference, Chicago, October 26-28, 2014.

\section{Special Recognitions and Awards/ Patents Issued}

1. J. Qu, Y. Zhou, D.N. Leonard, H.M. Meyer, H. Luo, ORNL Significant Event Award, "Discovery and fundamental understanding of incompatibility between diamond-like-carbon coatings and lubricant additives provide new insights for future materials development," 2015. 